\title{
The Novel Combination of Nitroxoline and PD-1 Blockade, Exerts a Potent Antitumor Effect in a Mouse Model of Prostate Cancer
}

\author{
Naijin $\mathrm{Xu}^{1 *}$, Linglong Huang ${ }^{2}$, Xiezhao $\mathrm{Li}^{1,3^{*}}$, Masami Watanabe ${ }^{1,4}$, Chaoming $\mathrm{Li}^{1,2}$, Abai Xu2 , Chunxiao \\ $\mathrm{Liu}^{2}$, Qiang Li ${ }^{5}$, Motoo Araki1 ${ }^{1}$ Koichiro Wada1, Yasutomo Nasu ${ }^{1,4,6}$, Peng Huang 1,2,6凶 \\ 1. Department of Urology, Okayama University Graduate School of Medicine, Dentistry and Pharmaceutical Sciences, Okayama, Japan \\ 2. Department of Urology, Zhujiang Hospital, Southern Medical University, Guangzhou; China \\ 3. Department of Urology, The $5^{\text {th }}$ Hospital of Guangzhou Medical University, Guangzhou; China \\ 4. Center for Innovative Clinical Medicine, Okayama University Hospital, Okayama, Japan \\ 5. Jiangsu Asieris Pharmaceuticals Co., Ltd., Taizhou, Jiangsu 225300, P.R. China \\ 6. Okayama Medical Innovation Center, Okayama University, Okayama, Japan \\ *These authors contributed to the work equally and should be regarded as co-first authors. \\ $\triangle$ Corresponding author: Peng Huang, Department of Urology, Okayama University Graduate School of Medicine, Dentistry and Pharmaceutical Sciences, \\ Okayama, Japan. Tel: + 81-86-235-7997, Fax: +81-86-235-7884, E-mail address: huangpeng509@gmail.com \\ (C) Ivyspring International Publisher. This is an open access article distributed under the terms of the Creative Commons Attribution (CC BY-NC) license \\ (https://creativecommons.org/licenses/by-nc/4.0/). See http://ivyspring.com/terms for full terms and conditions.
}

Received: 2018.12.13; Accepted: 2019.02.08; Published: 2019.03.09

\begin{abstract}
Programmed cell death protein 1 (PD-1) blockade is a promising therapeutic strategy against prostate cancer. Nitroxoline has been found to have effective anticancer properties in several cancer types. We investigated the efficacy of a combination therapy involving nitroxoline and PD-1 blockade in a prostate cancer mouse model. In our in vitro analysis, we found that nitroxoline inhibited the viability and proliferation of the mouse prostate cancer cell line RM9-Luc-PSA. Additionally, nitroxoline downregulated the expressions of phospho-PI3 kinase, phospho-Akt (Thr308), phospho-Akt (Ser473), phospho-GSK-3ß, Bcl-2, and Bcl-xL. Nitroxoline also downregulated programmed death-ligand 1 (PD-LI) expression levels in prostate cancer cell line and tumor tissue. In our murine prostate cancer orthotopic model, nitroxoline plus PD-1 blockade synergistically suppressed tumor growth when compared with nitroxoline or PD-1 blockade alone, leading to reductions in tumor weight, bioluminescence tumor signals, and serum prostate-specific antigen levels. Furthermore, fluorescence-activated cell sorting analysis showed that the combination strategy significantly enhanced antitumor immunity by increasing $\mathrm{CD} 44^{+} \mathrm{CD} 62 \mathrm{~L}^{+} \mathrm{CD} 8^{+}$ memory $T$ cell numbers and reducing myeloid-derived suppressor cell numbers in peripheral blood. In conclusion, our findings suggest that nitroxoline plus PD-1 blockade may be a promising treatment strategy in patients with prostate cancer.
\end{abstract}

Key words: prostate cancer, immunotherapy, chemotherapy, preclinical model, combination therapy

\section{Introduction}

Prostate cancer is the second most common malignant tumor in the male population worldwide [1]. The current standard treatment approach for patients involves surgery, chemotherapy, and radiation [2]. Recent studies confirmed that nitroxoline, an FDA-approved treatment for urinary tract infections, has anticancer activity against human prostate cancer [3], and immunotherapy might prolong patient survival in cases of multiple cancers [4].

Programmed cell death protein 1 (PD-1), a receptor expressed on the membrane of tumorinfiltrating lymphocytes, causes a series of changes, including the inhibition of CD8+ T cell viability and proliferation, reduction of cytokine production, and exhaustion of T cells [4]. PD-1 blockade is an effective therapeutic strategy for the treatment of several solid 
tumors, including prostate cancer [5-8]. Clinical trials on the treatment of metastatic castration-resistant prostate cancer have been initiated [9] (ClinicalTrials. gov: NCT02861573, NCT02499835, NCT03093428, NC T02787005, NCT02325557, and NCT02933255). PD-L1, a ligand of PD-1, is expressed on the surface of immune cells and many types of tumor cells. The interaction between PD-1 and PD-L1 suppresses the function of cytotoxic T lymphocytes (CTLs) [10]. PD-L1 expression is a predictive biomarker for development and prognosis in several cancer types [11-13]. Recent data showed that PD-L1 is highly expressed in prostate cancer and may be an indicator of biochemical recurrence. PD-L1 expression positively correlates with response rates in anti-PD-1or anti-PD-L1 therapy [14].

Nitroxoline has been found to exhibit anticancer activity against several cancers, including myeloma, breast cancer, glioma, bladder cancer, pancreatic cancer, and prostate cancer [3, 15-19]. The possible mechanisms underlying these anticancer effects involve the activation of cell apoptosis, arrest of the cell cycle, and suppression of angiogenesis through the inhibition of MetAP2 activity [15-18]. However, the direct immune response activity of nitroxoline has not yet been reported. A recent study reported that clioquinol, a close analog of nitroxoline, regulates $\mathrm{T}$ cell activation, proliferation, and apoptosis by inhibiting proteins related to NF-kB activation [20]. Other studies have shown that nitroxoline is an effective and reversible inhibitor of cathepsin B activity $[18,21]$. As an immune response mechanism, cathepsin B can inhibit lymphocyte proliferation and mediate immune cell apoptosis [22]. Additionally, nitroxoline exerts an antimicrobial effect through the chelation of iron and zinc, which serves as a regulator of immunity and is involved in the signal transduction of immune cells [23]. Therefore, we hypothesized that nitroxoline, which is associated with immune responses, may improve the efficacy of immune checkpoint inhibitor treatment.

The present study aimed to investigate the efficacy of a combination therapy involving nitroxoline and PD-1 blockade in a prostate cancer mouse model. The findings of this study might help in the development of more effective treatments for prostate cancer.

\section{Materials and Methods}

\section{Antibodies and reagents}

Nitroxoline treated with lysine salt was obtained from Jiangsu Asieris Pharmaceuticals, Co., Ltd. (Taizhou, Jiangsu, China). Nitroxoline and anti-mouse PD-1 (RMPI-14, BioXcell, West Lebanon, NH, USA) were dissolved in phosphate-buffered saline (PBS).
The following antibodies were used for Western blot analysis: CDK2 (\#2546, CST, Billerica, MA, USA), cyclin D3 (\#2936, CST), CDK6 (\#3136, CST), P53 (\#2524, CST), $\beta$-actin (\#4970, CST), cyclin-A (sc-596, Santa Cruz Biotechnology, Santa Cruz, CA, USA), phospho-Akt (Thr308) (\#4056, CST), phospho-Akt (Ser473) (\#4060, CST), PI3-kinase (\#4249, CST), phospho-PI3-kinase (\#4228, CST), phospho-GSK-3 $\beta$ (\#9323, CST), Bcl-2 (\#3498, CST), Bcl-xL (\#2762, CST), and cleaved caspase-3 (\#9664, CST). The following antibodies were used for immunohistochemistry and flow cytometry: Ki67 (\#12202, CST), CD31 (ab28364, Abcam, Cambridge, United Kingdom), FITC-labeled anti-CD11b antibody (553310, BD Bioscience, San Jose, CA, USA), PE-labeled anti-Gr-1 (553128, BD Bioscience), FITC-labeled anti-CD44 (11-0441-82, eBioscience, San Diego, CA, USA), PE-labeled anti-CD62L (120621-82, eBioscience), APC-CyTM7-labeled CD8 (5576 54, BD Bioscience), and appropriate FITC, PE, and APC-labeled isotype control antibodies. PD-L1 (17952-1-AP, Protein Tech Group, Chicago, USA) was used for Western blot, immunohistochemistry, and immunofluorescent staining. Goat anti-Rabbit IgG $(\mathrm{H}+\mathrm{L})$ Cross-Adsorbed Secondary Antibody, Alexa Fluor 488(\#A-11008, Thermo Fisher Scientific, Waltham, MA, USA) was used for immunofluorescent staining.

\section{Cell lines and cell culture conditions}

The human prostate cancer cell lines LNCaP, DU145, and PC3 and the normal epithelial prostate cell line RWPE-1 were purchased from ATCC (Manassas, VA, USA). LNCaP and DU145 cells were maintained in RPMI-1640 medium (Gibco, Invitrogen, Carlsbad, CA, USA) and DMEM (Gibco, Invitrogen) respectively. PC3 cells were maintained in Ham's F-12K (Kaighn's) medium (Gibco, Invitrogen). RWPE-1 cells were maintained in defined Keratinocyte-SFM liquid (Invitrogen). All cells were cultured at $37^{\circ} \mathrm{C}$ in a humidified atmosphere with $5 \% \mathrm{CO}_{2}$.

The murine prostate cancer cell line RM9 was kindly provided by Dr. T.C. Thompson (Baylor College of Medicine, Houston, TX, USA). RM9 cells were infected with plasmids containing the luciferase and human prostate-specific antigen (PSA) genes and named RM9-Luc-PSA cells. The new recombinant cell line was constructed and verified during our previous study [24]. The transfected RM9-Luc-PSA cells are ideally suited for real-time monitoring of drug responses and tumor progression. The cell line was cultured in DMEM supplemented with $10 \%$ fetal bovine serum and $1 \%$ penicillin/streptomycin.

\section{XTT viability assay}

Cell viability was measured using the XTT assay 
(Roche Diagnostics, Indianapolis, IN, USA). Cells $(1,000 /$ well $)$ were seeded in a 96-well plate and incubated overnight. Cells were then incubated with nitroxoline at concentrations ranging from 0 to $50 \mu \mathrm{M}$ for 24, 48, 72, and $96 \mathrm{~h}$. After incubation, XTT compound was added to each well and the mixture was incubated for $4 \mathrm{~h}$, as described previously [25].

\section{Colony-formation assay}

Cells (200/well) were seeded in a 6-well plate. After overnight attachment, the cells were exposed to the treatment for $48 \mathrm{~h}$. The medium was replaced every 3 days for approximately 14 days, at which time most of the colonies had more than 50 cells. The colonies were fixed with $4 \%$ paraformaldehyde, stained with $0.1 \%$ crystal violet, and finally counted by visual inspection.

\section{Cell cycle distribution analysis}

According to the instructions for the Cell Cycle Analysis kit (BD Biosciences, San Jose, CA, USA), cells were incubated in nitroxoline $(0,1,5$, and $10 \mu \mathrm{M})$ for $48 \mathrm{~h}$. Cells were then fixed with pre-cooled $70 \%$ ethanol overnight at $-20^{\circ} \mathrm{C}$. Subsequently, cells were washed twice to remove the ethanol (the first wash was in PBS and the second wash was in stain buffer [\#554656, BD Biosciences]) and were resuspended in PI/RNase staining buffer (\#550825, BD Biosciences). The cells were further incubated for $15 \mathrm{~min}$ and were analyzed using MACSQuant Analyzer 10 (Miltenyi Biotec, Cologne, Germany).

\section{Western blot analysis}

Cells were harvested and lysed in ice-cold lysis buffer that included a protease and phosphatase inhibitor cocktail (\#78410, Thermo Scientific, Waltham, MA, USA). Protein samples were separated by $10 \%$ SDS/polyacrylamide gel (Bio-Rad, Hercules, CA, USA) electrophoresis and were transferred to a polyvinylidene difluoride membrane. The membrane was incubated with primary antibodies overnight at $4^{\circ} \mathrm{C}$. Subsequently, the membrane was washed and incubated with HRP-secondary antibodies for $1 \mathrm{~h}$. The immunoblots were visualized using the enhanced chemiluminescence (ECL) kit (Amersham Pharmacia Biotech, Chandler, AZ, USA). $\beta$-actin was used as an internal reference.

\section{Orthotopic implantation of mouse prostate cancer cells into C57BL/6 mice}

Male C57BL/6 mice (aged 6-8 weeks) were obtained from Japan SLC, Inc. (Shizuoka, Japan) and were maintained in a specific-pathogen-free environment. All animal experiments were performed in accordance with the Guide of the Experimental Animals Center of Okayama University. Cells were harvested and resuspended in PBS at $1 \times 10^{5}$ cells/10 $\mu \mathrm{L}$. Each mouse was injected in the dorsolateral prostate with RM9-Luc-PSA cells for the orthotopic model. After tumor formation, mice were divided into the following groups: vehicle control (PBS), nitroxoline, PD-1 blockade, and nitroxoline + PD-1 blockade. Bioluminescence images were acquired and analyzed using the IVIS-200 Imaging System (Xenogen, Alameda, CA, USA).

\section{Flow cytometry analysis}

Blood samples from the posterior venous plexus of mouse eyeballs were collected into tubes containing EDTA. Samples were stained with primary antibodies for $1 \mathrm{~h}$ at $4^{\circ} \mathrm{C}$ and were washed twice with PBS. Stained samples were resuspended in $250 \mu \mathrm{L}$ of cold PBS and were analyzed using the MACSQuant Analyzer 10 (Miltenyi Biotec, Cologne, Germany).

\section{ELISA for serum PSA}

Serum PSA levels were measured with a human PSA ELISA kit (EL10005, ANOGEN, Ontario, Canada), according to the manufacturer's instructions. Serum $(50 \mu \mathrm{L})$ or standards were added to microtiter plates pre-coated with a monoclonal antibody specific for PSA. The optical density was measured at a wavelength of $450 \mathrm{~nm}$ using Microplate Manager 5.0 PC software (Bio-Rad, Hercules, CA, USA).

\section{Immunohistochemistry}

Tumor tissue was removed, fixed in formalin, and embedded in paraffin. After deparaffinization and rehydration of the tissue section, antigen retrieval was performed via autoclave treatment in $10-\mathrm{mM}$ sodium citrate buffer $\left(\mathrm{pH} \mathrm{6.0)}\right.$ for $20 \mathrm{~min}$ at $120^{\circ} \mathrm{C}$. Hydrogen peroxide (3\%) was used to block endogenous peroxidase for $10 \mathrm{~min}$ and the sections were placed in 5\% normal goat serum solution to prevent non-specific staining. Tissue sections were then incubated with the primary antibody in a humidified chamber overnight at $4^{\circ} \mathrm{C}$. The sections were rinsed in PBS and incubated with Simple Stain ${ }^{\mathrm{TM}}$ Mouse $\operatorname{MaxPo(R)~(414341F,~Nichirei~Bioscience,~Inc.,~}$ Tsukiji, Tokyo, Japan). For staining, DAB substrate kits (425312F, Nichirei Bioscience, Inc.) were used and the sections were lightly counterstained with hematoxylin.

\section{Immunofluorescence staining}

After deparaffinization and rehydration of the tissue section, antigen retrieval was performed as described in immunohistochemistry. Hydrogen peroxide $(3 \%)$ was used to block endogenous peroxidase for $10 \mathrm{~min}$, and the sections were placed in $5 \%$ normal goat serum solution to prevent non-specific staining. For cultured cell lines, cells 
were fixed with $4 \%$ formaldehyde for $15 \mathrm{~min}$ at room temperature, rinsed three times in PBS for $5 \mathrm{~min}$, and immunostained. Tissue sections or cells were then incubated with the primary antibody in a humidified chamber overnight at $4^{\circ} \mathrm{C}$. The sections were rinsed in PBS and incubated with fluorochrome-conjugated secondary antibody for $1 \mathrm{~h}$ at room temperature in dark conditions. Sections were covered with mounting medium with DAPI (Vector Laboratories, Inc., Burlingame, CA, USA).

\section{Statistical analysis}

All data are presented as mean \pm standard deviation (SD). One-way analysis of variance or Student's $t$-test was used to assess differences. All statistical analyses were performed using GraphPad Prism version 7 (GraphPad Prism Software Inc., San Diego, CA, USA). A $P$-value $<0.05$ was considered to indicate a statistically significant result.

\section{Results}

\section{Effects of nitroxoline on RM9-Luc-PSA cell viability and proliferation}

To evaluate the effects of nitroxoline (structural information is shown in Fig. 1A) on RM9-Luc-PSA cell viability and proliferation, we performed XTT and colony formation assays. As shown in Fig. 1B, treatment with $10-\mu \mathrm{M}$ nitroxoline significantly inhibited cell viability in the prostate cancer cell lines RM9-Luc-PSA, LNCaP, DU145, and PC3 in a time-dependent manner. No significant cytotoxicity was observed in the normal RWPE-1 cells. Nitroxoline reduced the viability of RM9-Luc-PSA cells in a dose and time-dependent manner (Fig. 1C).

Colony formation assays showed that nitroxoline significantly inhibited the colony-forming ability of RM9-Luc-PSA cells. Colony numbers were lower in the nitroxoline group in a dose-dependent manner $\left({ }^{*} P\right.$ $<0.05, \mathrm{~N}=4$, Fig. 1D and E). Overall, these results indicated that nitroxoline suppressed the viability and proliferation of prostate cancer cells but had no cytotoxic effect on normal RWPE-1 cells in vitro.

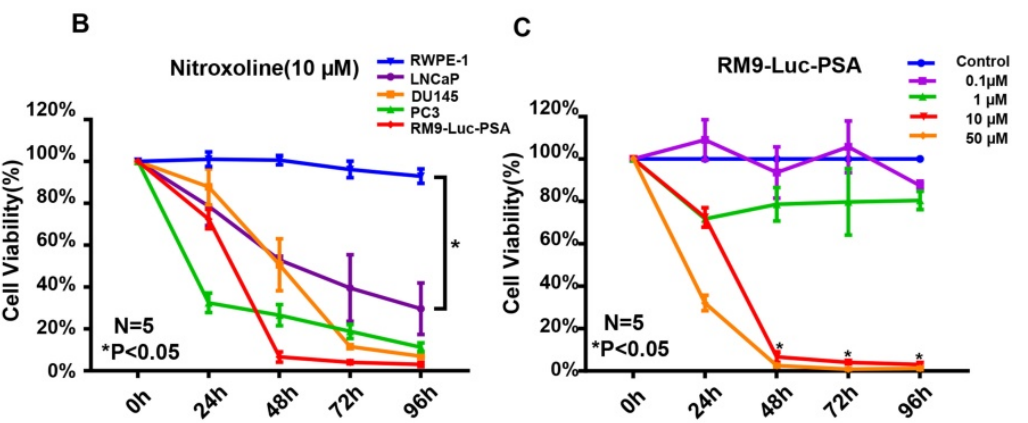

D

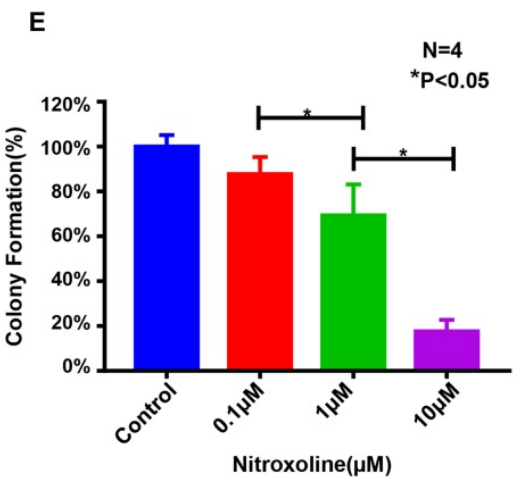

Figure 1. Nitroxoline suppresses the viability and colony-formation ability of RM9-Luc-PSA cells in vitro. (A) Chemical structure. Nitroxoline was treated with lysine salt to increase its solubility and stability in aqueous solution. (B) XTT assay of the normal immortalized cell line RWPE-1 and

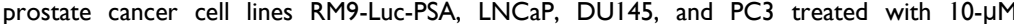
nitroxoline. Data are presented as mean \pm standard deviation $(N=5, * P<0.05)$. (C) Nitroxoline inhibited RM9-Luc-PSA cell growth in a dose- and time-dependent manner as shown by the XTT assay. Data are presented as mean \pm standard deviation $(N=5, * P<0.05$ vs.0.1 $\mathrm{MM})$. ( $\mathbf{D}$ and $\mathbf{E})$ RM9-Luc-PSA cells were incubated with increasing concentrations of nitroxoline for $48 \mathrm{~h}$, fixed, and stained after 14 days, and the number of colonies was counted under a microscope. Statistical analysis was performed for the colony numbers in all groups (control, $0.1 \mu \mathrm{M}, 1 \mu \mathrm{M}$, and $10 \mu \mathrm{M}$ ). Data are presented as mean \pm standard deviation $(\mathrm{N}=4$, $* P<0.05)$.

\section{Nitroxoline induced cell cycle arrest in RM9-Luc-PSA cells}

To explore whether nitroxoline regulates the cell cycle of prostate cancer cells, we evaluated the cell cycle distribution after treatment with various concentrations of nitroxoline for $48 \mathrm{~h}$. As shown in Fig. 2A, nitroxoline significantly increased the proportion of cells in the $S$ phase and reduced the proportion of cells in the G0/G1 phase (S, from $6.02 \pm 0.46 \%$ [control] to $23.29 \pm 1.97 \%$ [10 $\mu \mathrm{M}$ ]; G0/G1, from $47.99 \pm 0.91 \%$ [control] to $26.28 \pm$ $3.06 \%\left[\begin{array}{lll}10 \mu \mathrm{M} & \mathrm{H}\end{array}\right)$. We also evaluated the levels of cell cycle regulatory molecules, including CDK2, CDK6, cyclin-A, cyclin D3, and P53. Treatment with nitroxoline decreased the expressions of CDK2, CDK6, and cyclin D3 in a dose-dependent manner (Fig. 2B).

\section{Nitroxoline inhibited RM9-Luc-PSA cells through the PI3K/AKT pathway}

We investigated whether the PI3K/ AKT pathway is associated with the anticancer effect of nitroxoline. As shown in Fig. 3A, phospho-PI3 kinase, phosphoAkt (Thr308), phospho-Akt (Ser473), and phospho-GSK-3 $\beta$ were significantly decreased in RM9-Luc-PSA cells after 
treatment with nitroxoline. To further explore the mechanism of nitroxoline- induced cell apoptosis, the expressions of apoptosis- related proteins were assessed. We found that nitroxoline decreased Bcl-2 and Bcl-xL expressions and increased cleaved caspase-3 expression in RM9-Luc- PSA cells in a dose-dependent manner (Fig. 3B).

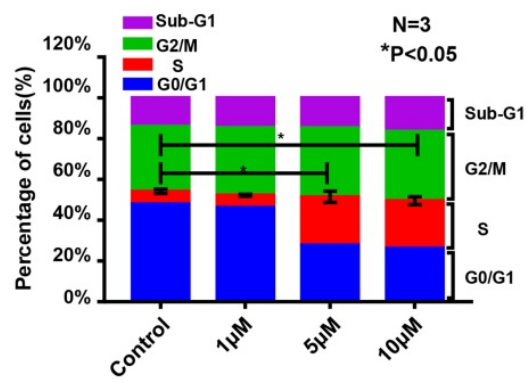

Nitroxoline $(\mu \mathrm{M})$
B

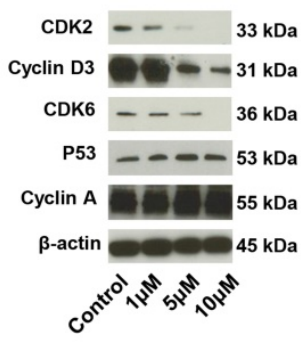

Nitroxoline $(\mu \mathrm{M})$
Figure 2. The cell cycle of RM9-Luc-PSA cells is affected by nitroxoline at different doses. (A) Cell cycle distributions in the different nitroxoline concentration groups (control, $1 \mu \mathrm{M}, 5 \mu \mathrm{M}$, and $10 \mu \mathrm{M}$ for $48 \mathrm{~h}$ ) according to flow cytometry analysis. Data are presented as mean \pm standard deviation $(\mathrm{N}=$ 3 , *P $<0.05$ ). (B) Expressions of cell cycle regulatory molecules, including CDK2, CDK6, cyclin-A, cyclin D3, and P53 in the different nitroxoline concentration groups (control, $1 \mu \mathrm{M}, 5 \mu \mathrm{M}$, and $10 \mu \mathrm{M}$ for $48 \mathrm{~h}$ ) according to Western blot analysis.

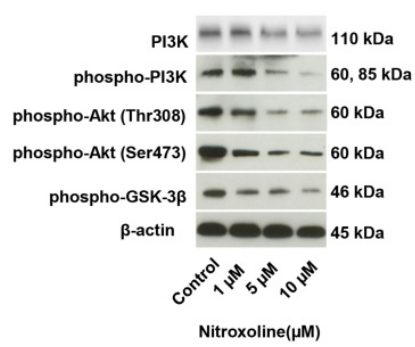

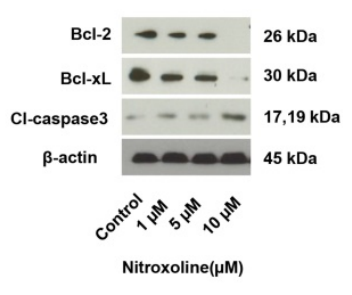

Figure 3. Western blot analysis for protein levels associated with the PI3K/AKT pathway and cancer cell apoptosis. (A) Expressions of PI3K/AKT pathway proteins, including PI3K, phospho-PI3 kinase, phospho-Akt (Thr308), phospho-Akt (Ser473), and phospho-GSK-3ß, in the different nitroxoline concentration groups (control, $1 \mu \mathrm{M}, 5 \mu \mathrm{M}$, and $10 \mu \mathrm{M}$ for $48 \mathrm{~h}$ ). (B) Expressions of apoptosis-related proteins, including Bcl-2, Bcl-xL, and cleaved caspase- 3 in the different nitroxoline concentration groups (control, $1 \mu \mathrm{M}, 5$ $\mu \mathrm{M}$, and $10 \mu \mathrm{M}$ for $48 \mathrm{~h}$ ). $\beta$-actin expression was used as an internal control.

\section{Nitroxoline suppressed PD-L1 expression in RM9-Luc-PSA cells and tumor tissue}

We examined PD-L1 expression in prostate cancer cell lines DU145, LNCaP, PC3, and RM9-LucPSA. In addition to PC3, all other cell lines expressed high levels of PD-L1 (Fig.4A). Similarly, Western blot analysis showed that PD-L1 was highly expressed in DU145, LNCaP, and RM9-Luc-PSA (Fig.4B). In RM9-Luc-PSA cells, nitroxoline significantly reduced
PD-L1 expression in a dose- and time-dependent manner (Fig. 4C). In tumor tissue specimens of the resected orthotopic tumor, the effect of nitroxoline on PD-L1 expression was assessed using immunohistochemistry (IHC) and immunofluorescent (IF) staining. PD-L1 was predominantly localized on the membrane or in the cytoplasm of the tumor cells and its expression level decreased after treatment with nitroxoline (15mg/kg) (Fig. 4D).

\section{Nitroxoline potentiated the antitumor effect of PD-1 blockade therapy in a murine orthotopic prostate tumor model}

In an orthotopic model of RM9-Luc-PSA prostate cancer, we explored the therapeutic potential of nitroxoline and PD-1 blockade. The experimental schedule is presented in Fig. 5A. PD-1 blockade was administered intraperitoneally at $200 \mu \mathrm{g}$ per mouse on days $0,4,8$, and 12 . Nitroxoline was intragastrically administered at $15 \mathrm{mg} / \mathrm{kg}$ per mouse on days $0,1,3$, $4,6,7,9,10,12$, and 13 five times a week. In vivo bioluminescence imaging data showed that nitroxoline was as effective as PD-1 blockade $(P<0.05$ when compared with control; $P>0.05$ when compared with PD-1 blockade alone) (Fig. 5B and C). Tumor size was significantly lower in the nitroxoline plus PD-1 blockade group than in the other groups ( $P$ $<$ 0.05). Furthermore, the tumor weight was significantly decreased in the nitroxoline plus PD-1 blockade group than in the nitroxoline or PD-1 blockade group alone $(P<0.05)$ (Fig. 5D and E).

We investigated histological sections of the resected orthotopic tumor to verify Ki-67 and CD31 expressions, which are used to assess cell proliferation and microvascular density (MVD), respectively. As shown in Fig.5F, there were fewer Ki-67-positive tumor cells in the nitroxoline plus PD-1 blockade group than in the monotherapy group. Moreover, MVD was significantly lower in the nitroxoline plus PD-1 blockade group than in the monotherapy group.

Our previous study showed that serum PSA levels were positively correlated with observed bioluminescence imaging findings [24]. In the present study, we found that the mean serum PSA level was lower in the nitroxoline plus PD-1 blockade group than in the control, nitroxoline, and PD-1 blockade groups $(P<0.05)$ (Fig. 5G).

\section{Nitroxoline combined with PD-1 blockade increased CD44+CD62 L+CD8+ memory $T$ cell numbers and reduced $C D 11 b^{+} \mathbf{G r}_{-1}{ }^{+}$ myeloid-derived suppressor cell (MDSC) numbers in the peripheral blood of tumor-bearing mice}

To examine the effect of nitroxoline + PD-1 
blockade, peripheral blood from C57BL/ 6 mice was assessed for $\mathrm{CD} 44^{+} \mathrm{CD} 62 \mathrm{~L}^{+} \mathrm{CD} 8{ }^{+}$memory $\mathrm{T}$ cells and $\mathrm{CD} 11 \mathrm{~b}^{+} \mathrm{Gr}-1^{+}$MDSCs. The proportions of memory $\mathrm{T}$ cells in the peripheral blood of mice from the control, nitroxoline, PD-1 blockade, and nitroxoline plus PD-1 blockade groups were $14.9 \pm 1.4 \%, 17.1 \pm 2.0 \%, 14.6 \pm$ $5.1 \%$, and $31.5 \pm 0.7 \%$, respectively. The proportions of memory $\mathrm{T}$ cells were significantly higher in the nitroxoline plus PD-1 blockade group than in the other groups $(P<0.05)(\mathrm{N}=3$; Fig. $6 \mathrm{~A}$ and $\mathrm{B})$. The proportions of MDSCs in the peripheral blood of mice from the control, nitroxoline, PD-1 blockade, and nitroxoline plus PD-1 blockade groups were $56.1 \pm$ $1.5 \%, 48.3 \pm 0.8 \%, 46.3 \pm 8.0 \%$, and $27.9 \pm 1.7 \%$, respectively. The proportion of MDSCs was significantly lower in the nitroxoline plus PD-1 blockade group than in the other groups $(P<0.05)(\mathrm{N}$ $=3$; Fig. $6 \mathrm{C}$ and D). Nitroxoline alone had no effect on the numbers of MDSCs and $\mathrm{CD} 44^{+} \mathrm{CD} 62 \mathrm{~L}^{+} \mathrm{CD} 8{ }^{+}$ memory T cells, but combined with PD-1 blockade, it caused an increase in the number of $\mathrm{CD}_{4}{ }^{+} \mathrm{CD} 62 \mathrm{~L}^{+} \mathrm{CD} 8{ }^{+}$memory $\mathrm{T}$ cells and a reduction in the number of MDSCs, suggesting that the combination therapy enhanced antitumor immunity.

\section{Discussions}

Immune checkpoint inhibitors, including CTLA-4 and PD-1 inhibitors, have shown enduring antitumor responses in preclinical and clinical research [26]. Recent evidence suggests that prostate cancer exhibits a limited response to PD-1 blockade monotherapy $[9,27]$. Numerous studies have focused on distinguishing the mechanisms of chemotherapy, cancer vaccines, immune-stimulatory agents, and small molecules to strengthen the efficacy of immune checkpoint therapy [28-31]. In this study, we found that the combination of nitroxoline and PD-1 blockade might be an effective alternative to improve the response rate of PD-1 blockade therapy.
A
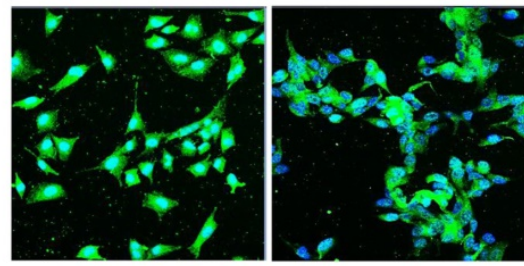

DU145

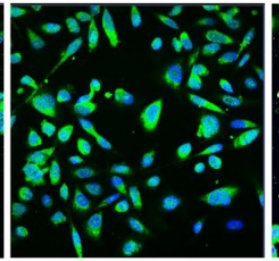

PC3

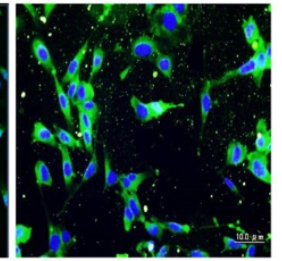

RM9-Luc-PSA

B

C
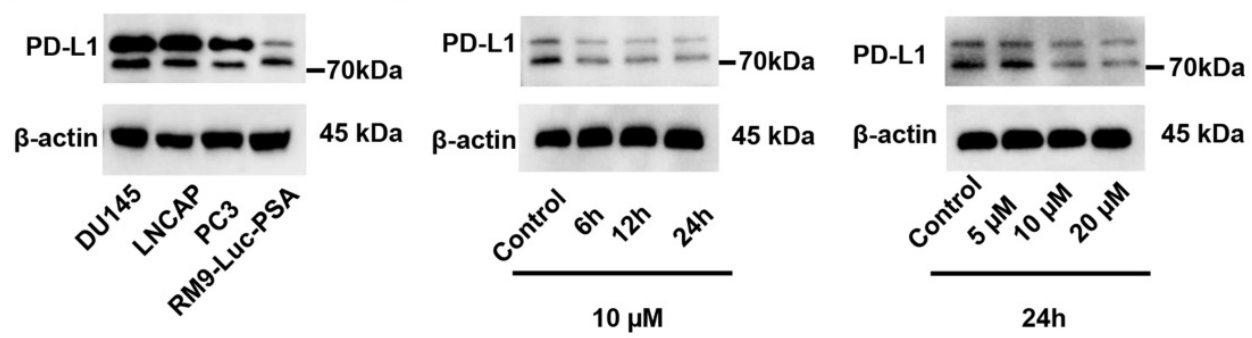

Control Nitroxoline Control Nitroxoline

D
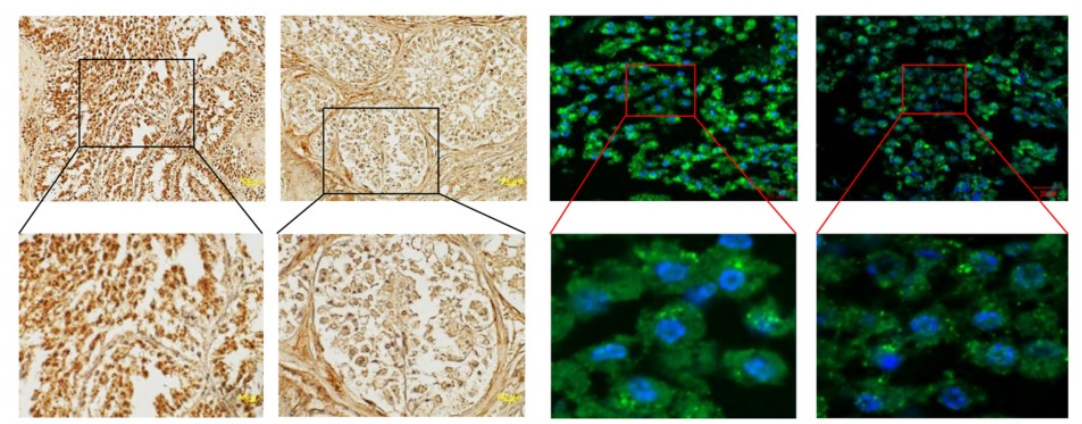

Figure 4. Nitroxoline suppresses PD-L1 expression in RM9-Luc-PSA cells and tumor tissue. (A) Expression of PD-L1 was detected in DU145, LNCaP, PC3, and RM9-Luc-PSA cells using immunofluorescent (IF) analysis. Green, staining for PD-L1; blue, nuclei staining with DAPI. Scale bar: $10 \mu \mathrm{m}$ (B) Expression of PD-L1 in prostate cancer cell lines by Western blot analysis. (C) Nitroxoline downregulated the expression of PD-L1 in RM9-Luc-PSA cells after treatment at different time points and concentrations. $\beta$-actin was used as an internal control. (D) Immunohistochemistry (IHC) and immunofluorescent (IF) staining of PD-LI in RM9-Luc-PSA prostate tumor tissue treated with or without nitroxoline $(15 \mathrm{mg} / \mathrm{kg})$. Scale bar of IHC, $100 \mu \mathrm{m}$; Scale bar of IF, $20 \mu \mathrm{m}$. 
A

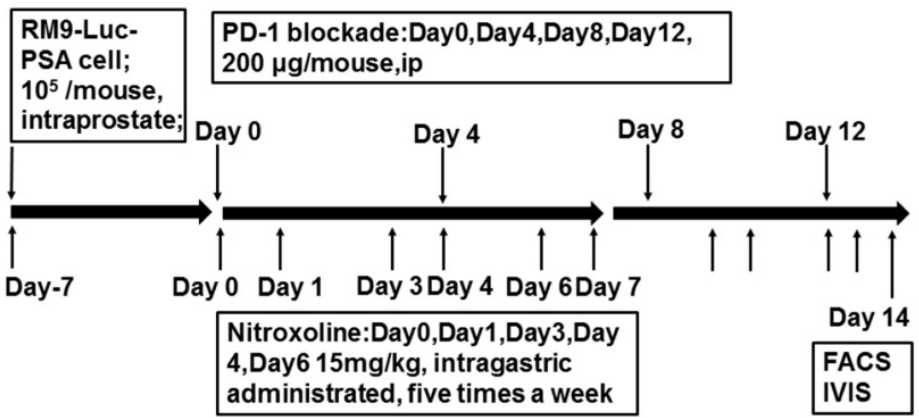

C

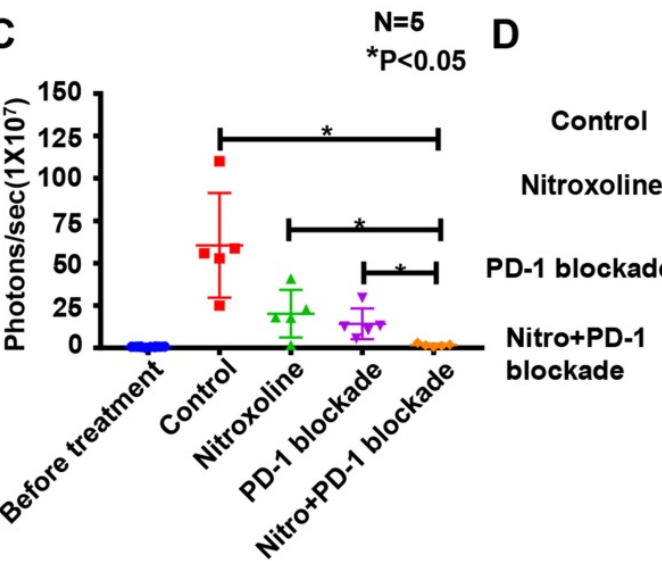

$\mathbf{F}$

K.67

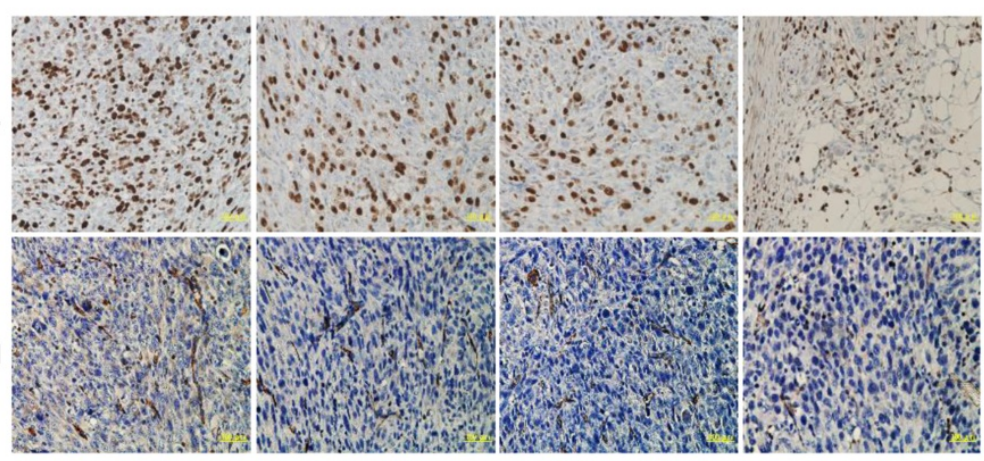

Control

Nitroxoline

PD-1 blockade Nitro+PD-1 blockade
B

\section{Before treatment}
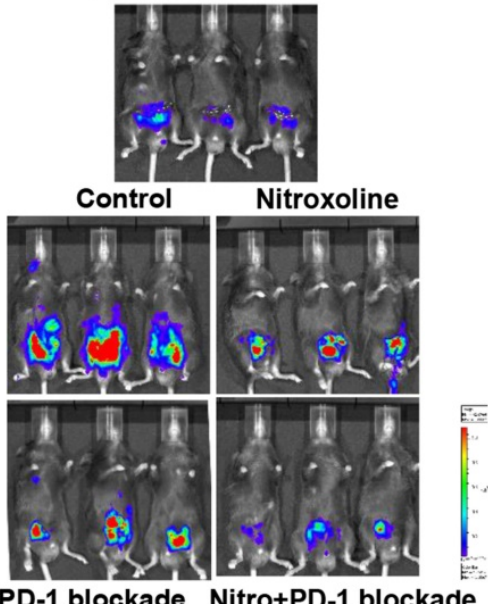

PD-1 blockade Nitro+PD-1 blockade

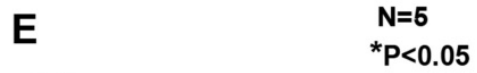

G
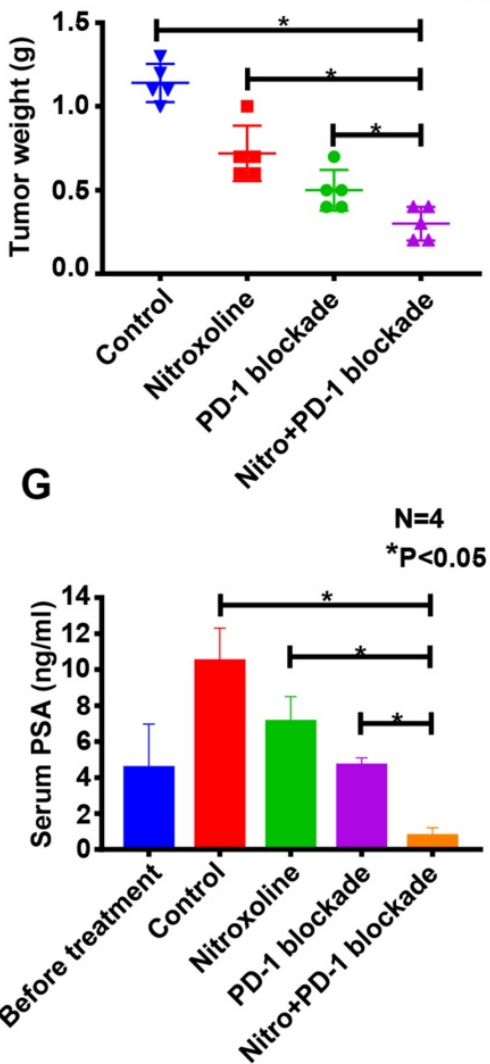

Figure 5. Nitroxoline inhibits tumor growth in the RM9-Luc-PSA orthotopic prostate cancer mouse model. (A) The therapeutic plan of nitroxoline or programmed cell death protein 1 (PD-1) blockade monotherapy and combined therapy in mice. (B) Bioluminescence images were evaluated to monitor tumor growth, using the IVIS imaging system. (C) The tumor-derived bioluminescent signal was measured by photons per second. Bioluminescence was quantified, and data are presented as mean \pm standard deviation $(\mathrm{N}=5, * \mathrm{P}<0.05)$. (D) Orthotopic prostate tumors were dissected, and representative images were provided. (E) A quantified graph of tumor weight at the experiment endpoint. Data are presented as mean \pm standard deviation $(N=5$, $* P<0.05)$. (F) Representative images of Ki-67 and $C D 31$ immunohistochemistry staining. Scale bar: $100 \mu \mathrm{m}(\mathbf{G})$ Serum prostate-specific antigen levels were significantly lower in the nitroxoline plus PD-1 blockade group than in the control, nitroxoline, and PD-1 blockade groups. Data are presented as mean \pm standard deviation $(\mathrm{N}=4, * P<0.05)$.

Nitroxoline has been used to treat urinary tract infections for over 50 years, and recently, it was considered as a potential anticancer agent against various cancer types [3, 15-19]. A phase II clinical trial in China (registration no. CTR20131716) has been conducted to assess the antitumor efficacy of nitroxoline in non-muscle invasive bladder cancer patients. Our study revealed that nitroxoline effectively suppressed the viability and proliferation of RM9-Luc-PSA cells in vitro. It has been reported that cell cycle progression in cancer cells is potentially associated with the irregular expression of cyclin/ 
CDK complexes [32]. Reduced expressions of cyclin D3, CDK2, and CDK6 in response to nitroxoline treatment induced cell cycle arrest at the $S$ phase, suggesting that nitroxoline changed the cell cycle distribution of RM9-Luc-PSA cells through cyclin/ CDK complexes. We further assessed the effects of nitroxoline on cell apoptosis and found that nitroxoline notably downregulated apoptosis-related proteins, including Bcl-2 and Bcl-xL. Both the Bcl-2 family and caspase family act as key factors in the apoptosis pathway [33]. Caspase-3, a major executive apoptotic enzyme, was found to be significantly activated after nitroxoline treatment on Western blot analysis, suggesting the occurrence of cell apoptosis. Similarly, some recent studies have shown that nitroxoline inhibites cancer cells, in part, by regulating proteins, including caspase- $3, \mathrm{PARP}, \mathrm{P} 53, \mathrm{Bcl}-\mathrm{xL}$, and Mcl-1 $[3,15,17]$. These results indicate that nitroxoline suppresses the proliferation of RM9-Luc-PSA cells through cell cycle arrest, activation of caspase-3, and regulation of Bcl-2 family proteins. In our previous studies, we revealed the synergistic effect of chemotherapy, including YM155 and lycorine, during immunotherapy $[25,34]$. Although no direct immune response associated with nitroxoline treatment has been reported until now, some evidence has indicated that nitroxoline and its analogs have the potential to influence host immunity [18, 20, 22, 23]. Therefore, in the current study, we explored the antitumor effects potentially underlying the combination of nitroxoline and PD-1 blockade therapy.
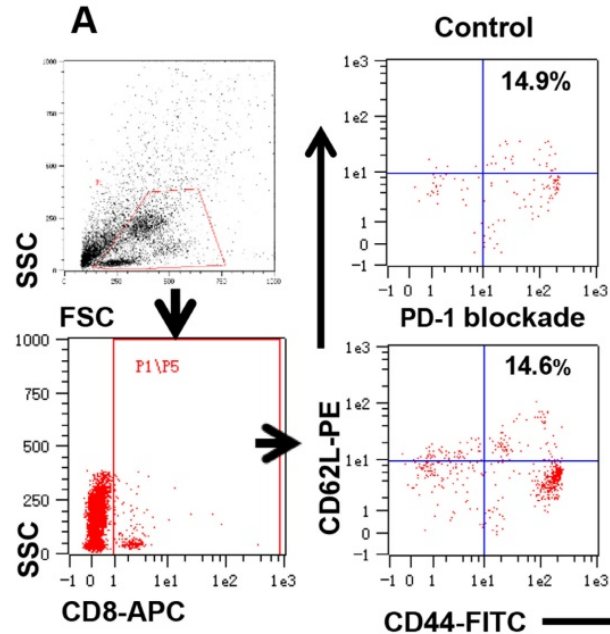

C

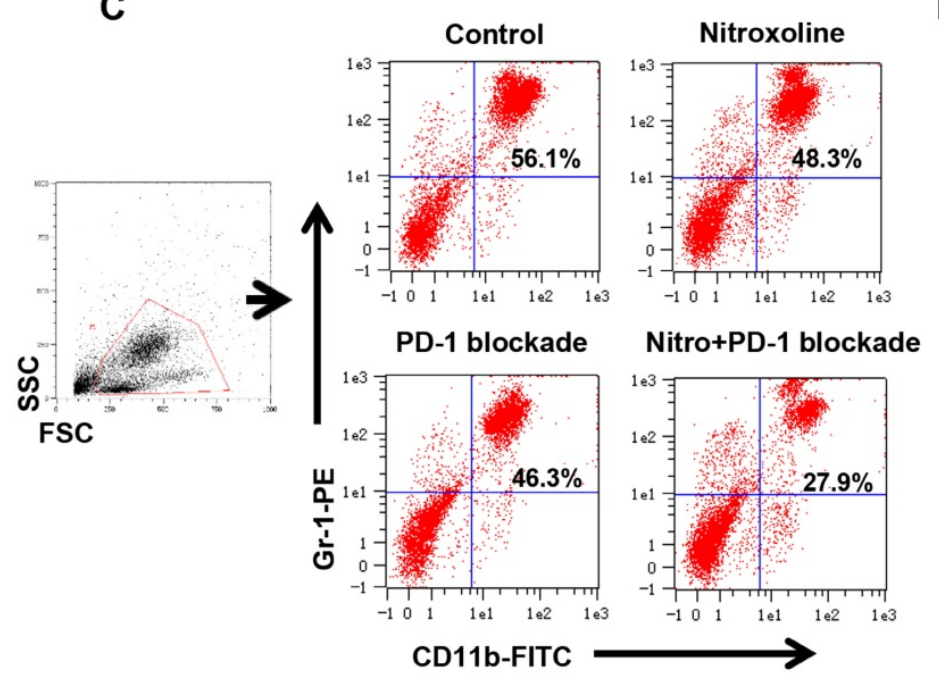

B
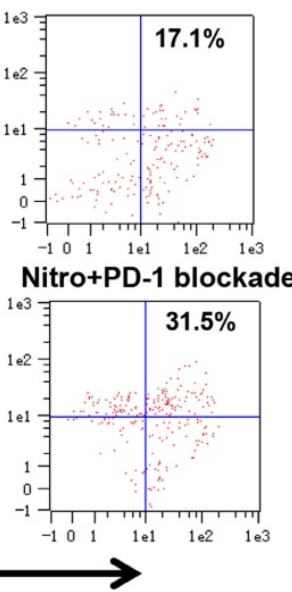

D
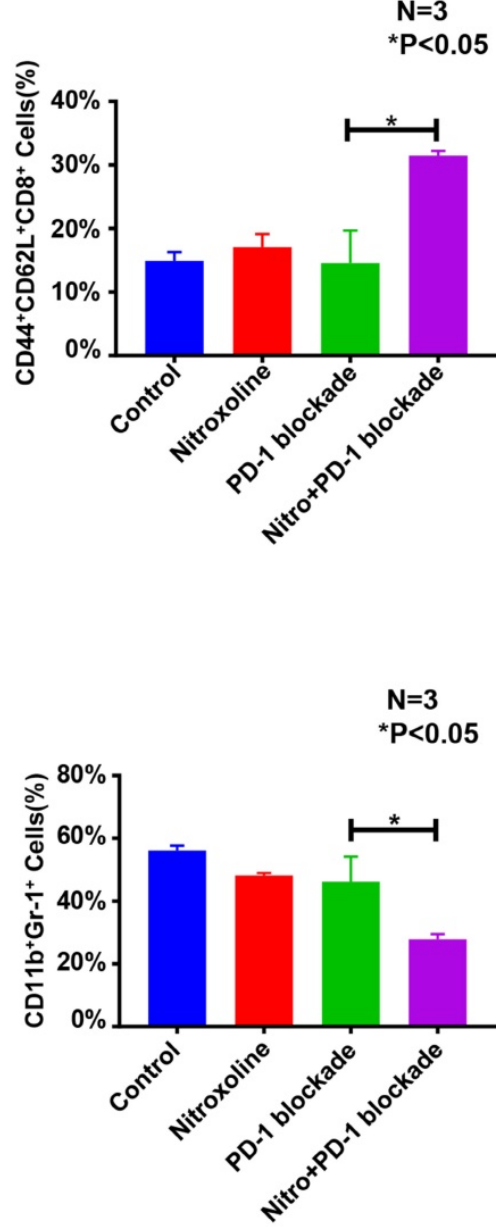

Figure 6. Nitroxoline combined with programmed cell death protein 1 (PD-1) blockade increases CD44+CD62L ${ }^{+C D} 8^{+}$memory $T$ cell numbers and reduces $\mathrm{CD} 1 \mathrm{lb}+\mathrm{Gr}-1^{+}$myeloid-derived suppressor cell (MDSC) numbers in peripheral blood. (A) Representative fluorescence-activated cell sorting (FACS) photographs of memory T cells from tumor-bearing mice at the experiment endpoint. (B) The percentage of CD44+CD62L $+C D 8^{+}$memory T cells was quantified by FACS analysis. A significant difference was observed between the nitroxoline plus PD-1 blockade group and the PD-1 blockade group. Data are presented as mean \pm standard deviation $(N=3, * P<0.05)$. (C) Representative FACS photographs of MDSCs. (D) The percentage of CD1 lb+Gr-1+ MDSCs was quantified by FACS analysis. A significant difference was observed between the nitroxoline plus PD-1 blockade group and the PD-1 blockade group. Data are presented as mean \pm standard deviation $(\mathrm{N}=3, * P<0.05)$. 
In the model of prostate cancer in vivo, we found that antitumor immunity was greater with nitroxoline plus PD-1 blockade than with nitroxoline or PD-1 blockade monotherapy, as evidenced by increased $\mathrm{CD} 44^{+} \mathrm{CD}^{2} \mathrm{~L}^{+} \mathrm{CD} 8{ }^{+}$memory $\mathrm{T}$ cell numbers and reduced MDSC numbers in peripheral blood. This result might be explained by several possible mechanisms. There is growing evidence suggesting that PI3K/AKT/mTOR inhibition can not only inhibit tumor cell proliferation, but also enhance tumor immune surveillance, which is accompanied by the secretion of immunosuppressive cytokines [35-37], the recruitment of MDSCs into the cancer region [38, 39] and the development of memory T cells [40]. AKT, a key component of the PI3K/AKT/mTOR pathway, controls the balance between terminal differentiation and memory T cell generation [41]. Additionally, AKT has been shown to significantly modulate the development and differentiation of MDSCs in vitro [42], which negatively regulates $\mathrm{T}$ cell function, leading to immune suppression [43-45]. We found that nitroxoline inhibited the expressions of important proteins in the PI3K/AKT/mTOR pathway, including phospho-PI3 kinase, phospho-Akt (Thr308), phosphoAkt (Ser473), and phospho-GSK-3 $\beta$. Although there is no direct evidence supporting the effects of nitroxoline on immune cell function and proliferation in this study, these factors, as discussed, might contribute to the mechanisms underlying increased $\mathrm{CD} 44^{+} \mathrm{CD}^{2} \mathrm{~L}^{+} \mathrm{CD} 8{ }^{+}$memory $\mathrm{T}$ cell numbers and reduced MDSC numbers in peripheral blood. PD-L1 expression indicates a poor prognosis and adverse clinicopathological outcomes and promotes tumor recurrence by inhibiting antitumor immunity $[12,13,46]$. PD-L1 is associated with higher response rates to anti-PD1/PD-L1 therapy, although positive response to treatment is also recorded for a lack of PD-L1 expression cases [47-49]. We found that nitroxoline downregulated the expression of PD-L1 at the cellular and tissue level; these results suggested that nitroxoline may block PD-L1 signaling and attenuate the interaction between PD-1 and PD-L1, ultimately enhancing the antitumor immune response. Taken together, the antitumor effects of nitroxoline combined with PD-1 blockade may be associated with the removal of immunosuppressive factors and the recovery of dysfunctional $\mathrm{T}$ cells. Further studies are necessary to clarify the specific mechanisms that underlie the immune response after nitroxoline treatment, as the current study only examined the immune response in peripheral blood. Furthermore, our research will focus on the effects of nitroxoline on the immune response with regard to several aspects, including the tumor environment, immune cell function, and energy metabolism.
To our knowledge, this is the first study to demonstrate that nitroxoline downregulates PD-L1 expression and inhibits tumor progression in prostate cancer. The combination of nitroxoline and PD-1 blockade increases the number of $\mathrm{CD} 44^{+} \mathrm{CD} 62 \mathrm{~L}^{+} \mathrm{CD} 8^{+}$ memory $\mathrm{T}$ cells and reduces immunosuppressive MDSCs. This appears to provide synergistic antitumor immunity in prostate cancer. Our results indicate that nitroxoline can enhance immunotherapy response.

In conclusion, nitroxoline plus PD-1 blockade is effective for prostate cancer and may serve as an alternative approach for treating prostate cancer. Considering that nitroxoline therapy and PD-1 blockade are approved by the FDA, our findings act as a basis for future studies on the use of these two approaches in combination for the treatment of patients with prostate cancer.

\section{Abbreviations}

PD-1: programmed cell death protein 1; PD-L1: programmed death-ligand 1; MDSCs: myeloidderived suppressor cells; ELISA: enzyme-linked immunosorbent assay; FDA: Food and Drug Administration; PSA: prostate specific antigen; FACS: fluorescence-activated cell sorting.

\section{Acknowledgements}

This study was funded by grants from the Ministry of Education, Culture, Sports, Science and Technology of Japan (No. 17K11138), the Science and Technology Planning Project of the Guangdong Province (2016A020215109), and the China Scholarship Council. We thank Mr. Hideo Ueki from Okayama University for his excellent technical assistance.

\section{Competing Interests}

The authors have declared that no competing interest exists.

\section{References}

1. Siegel RL, Miller KD, Jemal A. Cancer Statistics, 2017. CA Cancer J Clin. 2017; 67: 7-30.

2. Gomez-Veiga F, Rodriguez-Antolin A, Minana B, Hernandez C, Suarez JF, Fernandez-Gomez JM, et al. Diagnosis and treatment for clinically localized prostate cancer. Adherence to the European Association of Urology clinical guidelines in a nationwide population-based study - GESCAP group. Actas Urol Esp. 2017.

3. Chang WL, Hsu LC, Leu WJ, Chen CS, Guh JH. Repurposing of nitroxoline as a potential anticancer agent against human prostate cancer: a crucial role on AMPK/mTOR signaling pathway and the interplay with Chk2 activation. Oncotarget. 2015; 6: 39806-20

4. Pardoll DM. The blockade of immune checkpoints in cancer immunotherapy. Nat Rev Cancer. 2012; 12: 252-64.

5. Brahmer J, Reckamp KL, Baas P, Crino L, Eberhardt WE, Poddubskaya E, et al. Nivolumab versus Docetaxel in Advanced Squamous-Cell Non-Small-Cell Lung Cancer. N Engl J Med. 2015; 373: 123-35.

6. Motzer RJ, Escudier B, McDermott DF, George S, Hammers HJ, Srinivas S, et al. Nivolumab versus Everolimus in Advanced Renal-Cell Carcinoma. N Engl J Med. 2015; 373: 1803-13. 
7. Ferris RL, Blumenschein G, Jr., Fayette J, Guigay J, Colevas AD, Licitra L, et al. Nivolumab for Recurrent Squamous-Cell Carcinoma of the Head and Neck. N Engl J Med. 2016; 375: 1856-67.

8. Goswami S, Aparicio A, Subudhi SK. Immune Checkpoint Therapies in Prostate Cancer. Cancer J. 2016; 22: 117-20.

9. Isaacsson Velho P, Antonarakis ES. PD-1/PD-L1 pathway inhibitors in advanced prostate cancer. Expert Rev Clin Pharmacol. 2018; 11: 475-86,

10. Zou WP, Wolchok JD, Chen LP. PD-L1 (B7-H1) and PD-1 pathway blockade for cancer therapy: Mechanisms, response biomarkers, and combinations. Sci Transl Med. 2016; 8 .

11. Thompson RH, Kuntz SM, Leibovich BC, Dong HD, Lohse CM, Webster WS, et al. Tumor B7-H1 is associated with poor prognosis in renal cell carcinoma patients with long-term follow-up. Cancer Res. 2006; 66: 3381-5.

12. Lyford-Pike S, Peng SW, Young GD, Taube JM, Westra WH, Akpeng B, et al. Evidence for a Role of the PD-1:PD-L1 Pathway in Immune Resistance of HPV-Associated Head and Neck Squamous Cell Carcinoma. Cancer Res. 2013; 73: 1733-41.

13. Nakanishi I, Wada Y, Matsumoto K, Azuma M, Kikuchi K, Ueda S. Overexpression of B7-H1 (PD-L1) significantly associates with tumor grade and postoperative prognosis in human urothelial cancers. Cancer Immunol Immun 2007: 56: 1173-82.

14. Gevensleben H, Dietrich D, Golletz C, Steiner S, Jung M, Thiesler T, et al. The Immune Checkpoint Regulator PD-L1 Is Highly Expressed in Aggressive Primary Prostate Cancer. Clin Cancer Res. 2016; 22: 1969-77.

15. Jiang $\mathrm{H}$, Taggart JE, Zhang $X$, Benbrook DM, Lind SE, Ding WQ. Nitroxoline (8-hydroxy-5-nitroquinoline) is more a potent anti-cancer agent than clioquinol (5-chloro-7-iodo-8-quinoline). Cancer Lett. 2011; 312: 11-7.

16. Lazovic J, Guo L, Nakashima J, Mirsadraei L, Yong W, Kim HJ, et al. Nitroxoline induces apoptosis and slows glioma growth in vivo. Neuro Oncol. 2015; 17: 53-62.

17. Mao H, Du Y, Zhang Z, Cao B, Zhao J, Zhou H, et al. Nitroxoline shows antimyeloma activity by targeting the TRIM25/p53 axle. Anticancer Drugs. 2017; 28: 376-83

18. Mirkovic B, Markelc B, Butinar M, Mitrovic A, Sosic I, Gobec S, et al. Nitroxoline impairs tumor progression in vitro and in vivo by regulating cathepsin B activity. Oncotarget. 2015; 6: 19027-42.

19. Shim JS, Matsui Y, Bhat S, Nacev BA, Xu J, Bhang HE, et al. Effect of nitroxoline on angiogenesis and growth of human bladder cancer. J Natl Cancer Inst. 2010; 102: 1855-73.

20. Liang Y, Mao XL, Liu HY. Proteasome inhibitor clioquinol as a candidate drug in prophylaxis and treatment of acute graft-versus-host disease. Med Hypotheses. 2011; 76: 400-2.

21. Kos J, Mitrovic A, Mirkovic B. The current stage of cathepsin B inhibitors as potential anticancer agents. Future Med Chem. 2014; 6: 1355-71.

22. Conus S, Simon HU. Cathepsins and their involvement in immune responses. Swiss Med Wkly. 2010; 140: 4-11.

23. Haase H, Rink L. Zinc signals and immune function. Biofactors. 2014; 40: $27-40$

24. Xu P, Xu N, Guo K, Xu A, Takenaka F, Matsuura E, et al. Real-time monitoring of tumor progression and drug responses in a preclinical mouse model of prostate cancer. Oncotarget. 2016; 7: 33025-34.

25. Guo $\mathrm{K}$, Huang $\mathrm{P}, \mathrm{Xu} \mathrm{N}, \mathrm{Xu} \mathrm{P}, \mathrm{Kaku} \mathrm{H}$, Zheng $\mathrm{S}$, et al. A combination of YM-155, a small molecule survivin inhibitor, and IL-2 potently suppresses renal cell carcinoma in murine model. Oncotarget. 2015; 6: 21137-47.

26. Sharma P, Allison JP. Immune checkpoint targeting in cancer therapy: toward combination strategies with curative potential. Cell. 2015; 161: 205-14.

27. Cordes LM, Gulley JL, Madan RA. Perspectives on the clinical development of immunotherapy in prostate cancer. Asian J Androl. 2018; 20: 253-9.

28. Champiat S, Ileana E, Giaccone G, Besse B, Mountzios G, Eggermont A, et al. Incorporating immune-checkpoint inhibitors into systemic therapy of NSCLC. J Thorac Oncol. 2014; 9: 144-53.

29. Kim T, Amaria RN, Spencer C, Reuben A, Cooper ZA, Wargo JA. Combining targeted therapy and immune checkpoint inhibitors in the treatment of metastatic melanoma. Cancer Biol Med. 2014; 11: 237-46.

30. Pfirschke C, Engblom C, Rickelt S, Cortez-Retamozo V, Garris C, Pucci F, et al. Immunogenic Chemotherapy Sensitizes Tumors to Checkpoint Blockade Therapy. Immunity. 2016; 44: 343-54.

31. Mahoney KM, Rennert PD, Freeman GJ. Combination cancer immunotherapy and new immunomodulatory targets. Nat Rev Drug Discov. 2015; 14: 561-84.

32. Grana X, Reddy EP. Cell cycle control in mammalian cells: role of cyclins, cyclin dependent kinases (CDKs), growth suppressor genes and cyclin-dependent kinase inhibitors (CKIs). Oncogene. 1995; 11: 211-9.

33. Nunez G, Benedict MA, Hu Y, Inohara N. Caspases: the proteases of the apoptotic pathway. Oncogene. 1998; 17: 3237-45.

34. Li X, Xu P, Wang C, Xu N, Xu A, Xu Y, et al. Synergistic effects of the immune checkpoint inhibitor CTLA-4 combined with the growth inhibitor lycorine in a mouse model of renal cell carcinoma. Oncotarget. 2017; 8: 21177-86.

35. Ying HQ, Elpek KG, Vinjamoori A, Zimmerman SM, Chu GC, Yan HY, et al. PTEN Is a Major Tumor Suppressor in Pancreatic Ductal Adenocarcinoma and Regulates an NF-kappa B-Cytokine Network. Cancer Discov. 2011; 1: 158-69.

36. Dong Y, Richards JAE, Gupta R, Aung PP, Emley A, Kluger Y, et al. PTEN functions as a melanoma tumor suppressor by promoting host immune response. Oncogene. 2014; 33: 4632-42.
37. Peng W, Chen JQ, Liu C, Malu S, Creasy C, Tetzlaff MT, et al. Loss of PTEN Promotes Resistance to T Cell-Mediated Immunotherapy. Cancer Discov. 2016; 6: 202-16.

38. Schmid MC, Avraamides CI, Dippold HC, Franco I, Foubert P, Ellies LG, et al. Receptor tyrosine kinases and TLR/IL1Rs unexpectedly activate myeloid cell PI3kgamma, a single convergent point promoting tumor inflammation and progression. Cancer Cell. 2011; 19: 715-27.

39. Hirsch E, Ciraolo E, Franco I, Ghigo A, Martin M. PI3K in cancer-stroma interactions: bad in seed and ugly in soil. Oncogene. 2014; 33: 3083-90.

40. Ribas A, Shin DS, Zaretsky J, Frederiksen J, Cornish A, Avramis E, et al. PD-1 Blockade Expands Intratumoral Memory $\mathrm{T}$ Cells. Cancer Immunology Research. 2016; 4: 194-203.

41. Kim EH, Suresh M. Role of PI3K/Akt signaling in memory CD8 T cell differentiation. Front Immunol. 2013; 4: 20

42. Gato-Canas M, de Morentin XM, Blanco-Luquin I, Fernandez-Irigoyen J, Zudaire I, Liechtenstein $\mathrm{T}$, et al. A core of kinase-regulated interactomes defines the neoplastic MDSC lineage. Oncotarget. 2015; 6: 27160-75.

43. Ostrand-Rosenberg S, Sinha P, Beury DW, Clements VK. Cross-talk between myeloid-derived suppressor cells (MDSC), macrophages, and dendritic cells enhances tumor-induced immune suppression. Semin Cancer Biol. 2012; 22: 275-81.

44. Finke J, Ko J, Rini B, Rayman P, Ireland J, Cohen P. MDSC as a mechanism of tumor escape from sunitinib mediated anti-angiogenic therapy. Int Immunopharmacol. 2011; 11: 856-61.

45. Meyer C, Cagnon L, Costa-Nunes CM, Baumgaertner P, Montandon N, Leyvraz L, et al. Frequencies of circulating MDSC correlate with clinical outcome of melanoma patients treated with ipilimumab. Cancer Immunol Immunother. 2014; 63: 247-57.

46. Zhang Y, Huang SD, Gong DJ, Qin YH, Shen QA. Programmed death-1 upregulation is correlated with dysfunction of tumor-infiltrating CD8(+) T lymphocytes in human non-small cell lung cancer. Cell Mol Immunol. 2010; 7: 389-95.

47. Rizvi NA, Chow LQM, Dirix LY, Gettinger SN, Gordon MS, Kabbinavar FF, et al. Clinical trials of MPDL3280A (anti-PDL1) in patients (pts) with non-small cell lung cancer (NSCLC). Journal of Clinical Oncology. 2014; 32

48. Powles T, Eder JP, Fine GD, Braiteh FS, Loriot Y, Cruz C, et al. MPDL3280A (anti-PD-L1) treatment leads to clinical activity in metastatic bladder cancer. Nature. 2014; 515: 558-+

49. Cho DC, Sosman JA, Sznol M, Gordon MS, Hollebecque A, Hamid O, et al. Clinical activity, safety, and biomarkers of MPDL3280A, an engineered PD-L1 antibody in patients with metastatic renal cell carcinoma (mRCC). Journal of Clinical Oncology. 2013; 31. 